\title{
Spectral characterisation of high temperature solar absorber coatings
}

\author{
Leslie Herding ${ }^{1}$, Simon Caron ${ }^{2}$, a) , Volker Nickich ${ }^{1}$, Florian Sutter ${ }^{2}$ \\ ${ }^{1}$ TH Köln, CIRE, Betzdorfer Straße 2, 50679 Cologne, Germany \\ ${ }^{2}$ German Aerospace Center (DLR), Institute of Solar Research, Plataforma Solar de Almeria (PSA), 04200 Spain \\ a) corresponding author: $\underline{\text { simon.caron@dlr.de }}$
}

\begin{abstract}
Four newly developed solar absorber coatings are to be evaluated with the help of thermal cycling and optical characterisation. An initial round robin campaign reveals the need of a standardisation of the calculation procedure of solar absorptance and thermal emittance derived from optical measurements at room temperature. The implementation of a common processing of spectral hemispherical reflectance values enables a reduction of standard deviations to $0.2 \%$, both for solar absorptance and thermal emittance. The calculation procedure proposed within this paper corresponds to DIN EN 22975-3 and enables the verification of measurement reproducibility according to ASTM E $691-05$.
\end{abstract}

\section{INTRODUCTION}

Within the Horizon 2020 programme, the European Union launched the project Raiselife. The project "focuses on raising the lifetime" of key materials for Concentrated Solar Power (CSP) technology [1], [2]. It targets central receiver for tower installations. New materials and coatings to be used in Solar Tower (ST) applications are being developed and evaluated with the aim of improving thermal efficiency and durability while decreasing costs. Four new absorber coatings are tested at a dish facility at the joint CIEMAT (Centro de Investigaciones Energéticas, Medioambientales y Tecnológicas, Centre of energetic, environmental and technological investigation) and DLR (Deutsches Zentrum für Luft- und Raumfahrt, German Aerospace Center) investigation site Plataforma Solar de Almería (PSA) in Tabernas, southern Spain. The specimens are exposed to concentrated solar flux at skin temperatures up to $650{ }^{\circ} \mathrm{C}$ to investigate their failure mechanisms and optical characterisation is carried out. A round robin campaign is performed to verify the reproducibility of laboratory optical measurements carried out by the different project participants.

\section{OPTICAL CHARACTERISATION}

\section{Basic Equations}

For the evaluation of the coatings, spectral hemispherical reflectance values measured with two complementary spectrophotometers in the laboratory at room temperature are used to calculate two key factors: solar absorptance and thermal emittance. They allow the determination of the opto-thermal efficiency of the coatings.

\section{Solar Absorptance}

Solar absorptance $\left(\alpha_{s}\right)$ represents an indicator of the proportion of solar radiation being absorbed by the material in question. It is calculated as shown in Eq. 1 [3]. $\rho_{\text {hem }}$ denominates the spectral hemispherical reflectance at the wavelength $\lambda(\mathrm{nm})$ while $\mathrm{G}_{\text {sol }}$ represents the reference spectrum for solar direct normal irradiance $(\mathrm{W} / \mathrm{m} 2 . \mathrm{nm})$. 


$$
\alpha_{s}=\frac{\int_{\lambda 1}^{\lambda 2}\left[1-\rho_{\text {hem }}(\lambda)\right] \cdot G_{\text {sol }}(\lambda) \cdot d \lambda}{\int_{\lambda 1}^{\lambda 2} G_{\text {sol }}(\lambda) \cdot d \lambda}
$$

As reference spectrum, terrestrial direct and circumsolar solar irradiance at an Air Mass (AM) 1.5 is defined, according to ASTM G173-03 [4].

\section{Thermal Emittance}

Thermal emittance $\left(\varepsilon_{\mathrm{th}}\right)$ describes the fraction of black body exitance emitted by the absorber at the temperature in question. Hence, it is calculated weighting the spectral hemispherical reflectance $\rho_{\text {hem }}$ at the wavelength $\lambda(\mathrm{nm})$ with the ideal black body exitance $\mathrm{E}_{\mathrm{BB}}(\mathrm{W} / \mathrm{m} 2 . \mathrm{nm})$ representing the reference spectrum such as formulated in Eq. 2 [3].

$$
\varepsilon_{t h}(T)=\frac{\int_{\lambda 1}^{\lambda 3}\left[1-\rho_{h e m}(\lambda)\right] \cdot E_{B B}(\lambda, T) \cdot d \lambda}{\int_{\lambda 1}^{\lambda 3} E_{B B}(\lambda, T) \cdot d \lambda}
$$

In contrast to solar absorptance, thermal emittance is a temperature dependent indicator. This is due to the reference spectrum calculated according to Planck's Law as in Eq. 3 [5]. The relevant temperature is the skin temperature during thermal cycling, i.e.the reference is set at $923 \mathrm{~K}\left(650^{\circ} \mathrm{C}\right)$.

$$
E_{B B}(\lambda, T)=\frac{2 \cdot \pi \cdot h \cdot c^{2}}{\lambda^{5} \cdot\left[\exp \left(\frac{h \cdot c}{\lambda \cdot k \cdot T}\right)-1\right]}
$$

The exitance of an ideal black body, $\mathrm{E}_{\mathrm{BB}}$, depends on the wavelength $\lambda(\mathrm{nm})$ and the temperature $\mathrm{T}(\mathrm{K})$. Physical constants used are the Planck constant (h), speed of light (c) and the Boltzmann constant (k). These values are defined according to CODATA 2014 [6].

\section{Opto-Thermal Efficiency}

The variables defined in the laboratory are used to determine the opto-thermal efficiency $\eta_{\text {coating }}$ as expressed in Eq. 4 [3]. For the calculation, indicators of the conditions during field tests are included in the form of the incident solar flux $\mathrm{Q}_{\text {sol }}\left(\mathrm{W} / \mathrm{m}^{2}\right)$ and the skin temperature of the specimen $\mathrm{T}_{\mathrm{abs}}(\mathrm{K})$. $\sigma$ corresponds to Stefan Boltzmann's universal physical constant and is defined according to CODATA 2014 [6].

$$
\eta_{\text {coating }}=\frac{\alpha_{s} \cdot Q_{\text {sol }}-\varepsilon_{t h \cdot} \cdot \sigma \cdot T_{a b s}{ }^{4}}{Q_{\text {sol }}}
$$

\section{Round Robin Campaign}

For the evaluation of the coatings, optical characterisation is carried out in different laboratories. A flat specimen coated with a selective absorber coating has been measured at room temperature. A so-called round robin campaign is performed to assess the measurements. It aims at evaluating the reproducibility of the measurements, which is defined as "precision under reproducibility conditions" meaning "conditions where test results are obtained with the same method on identical test items in different laboratories with different operators using different equipment" [7]. 


\section{Equipment}

Table 1 shows the instruments used by the round robin participants for the determination of solar absorptance. They cover the ultraviolet, visible and near infrared (UV-VIS-NIR) spectral range.

Table 1 - List of instruments used by the round robin participants for the determination of solar absorptance [8]

\begin{tabular}{|c|c|c|c|c|c|}
\hline Participant & CT & IE & CS & $\begin{array}{c}\text { DLR/ } \\
\text { CIEMAT }\end{array}$ & IA \\
\hline Spectrophotometer & $\begin{array}{c}\text { Lambda 950, } \\
\text { Perkin Elmer }\end{array}$ & $\begin{array}{c}\text { Bruker } \\
\text { VERTEX 80 }\end{array}$ & $\begin{array}{c}\text { Lambda 950, } \\
\text { Perkin Elmer }\end{array}$ & $\begin{array}{c}\text { Lambda 1050, } \\
\text { Perkin Elmer }\end{array}$ & Cary 500 \\
\hline \begin{tabular}{c} 
Reference spectrum \\
\hline $\begin{array}{c}\text { Wavelength } \\
\text { range (nm) }\end{array}$
\end{tabular} & 250 to 2500 & 320 to 2400 & 250 to 2500 & 280 to 2500 & $\begin{array}{c}\text { ASTM E-409 } \\
\text { (AM0) }\end{array}$ \\
\hline
\end{tabular}

The table reveals that two participants (CT, CS) use the same instrument and one (DLR/CIEMAT) a later version of the same instrument. Wavelength ranges in consideration vary from a lower limit of $250 \mathrm{~nm}$ to $320 \mathrm{~nm}$ while all instruments provide spectral data up to 2,500 nm. All institutes but IA use the ASTM G173.03 AM1.5 direct reference spectrum while IA uses the ASTM E-409 AM0 spectrum.

Table 2 provides an overview of the instruments used for the determination of thermal emittance by the different institutes participating in the round robin campaign.

Table 2 - List of instruments used by the round robin participants for the determination of thermal emittance [8]

\begin{tabular}{|c|c|c|c|c|c|}
\hline Participant & CT & IE & CS & $\begin{array}{c}\text { DLR/ } \\
\text { CIEMAT }\end{array}$ & IA \\
\hline Spectrophotometer & $\begin{array}{c}\text { Frontier FT-IR, } \\
\text { Perkin Elmer }\end{array}$ & $\begin{array}{c}\text { Bruker } \\
\text { VERTEX } 80\end{array}$ & $\begin{array}{c}\text { SOC-100 } \\
\text { HDR, Surface } \\
\text { Optics } \\
\text { + Thermo- } \\
\text { electron } \\
\text { (Nicolet } \\
6700)\end{array}$ & $\begin{array}{c}\text { Frontier FT-IR, } \\
\text { Perkin Elmer }\end{array}$ & $\begin{array}{c}\text { Temp 2000A of } \\
\text { AZ Technology }\end{array}$ \\
\hline $\begin{array}{c}\text { Reference } \\
\text { spectrum }\end{array}$ & Planck's Law for black body radiation (at 923 K) & $\begin{array}{c}\text { Spectrum of the } \\
300 \mathrm{~K} \text { black } \\
\text { body }\end{array}$ \\
\hline $\begin{array}{c}\text { Wavelength } \\
\text { range }(\boldsymbol{\mu} \mathrm{m})\end{array}$ & 2 to 16 & 1.5 to 16 & 1.5 to 25 & 2 to 16 & 3 to 30 \\
\hline
\end{tabular}

The table reveals that only CT and DLR/CIEMAT use the same instrument. IA uses a portable device that calculates thermal emittance directly and does not provide spectral hemispherical reflectance data. Hence, only the initial value is included in the evaluation. All institutes but IA use Planck's Law for black body radiation at $923 \mathrm{~K}$ as reference spectrum while IA value is calculated for a blackbody at room temperature. Measured upper wavelength limits $\lambda_{3}$ range between 16 (CT, IE, DLR/CIEMAT) and 25-30 (CS, IA) $\mu \mathrm{m}$.

The analysis of initial DLR/CIEMAT data in the infrared (IR) range reveals a measurement deviation. Figure 1 (left) 
shows a mismatch of the two complementary spectrophotometers used at PSA. Lambda 1050 describes the equipment used for measurements up to 2,500 nm and Frontier FTIR the device depicted in Table 2. The raw measurements of the Frontier FTIR device deviate around $10 \%$ from the Lambda 1050 calibrated measurements in the common measurement range from 2,000 to 2,500 nm. Figure 1 (right) shows a comparison of the initial Frontier FTIR signal and the spectral hemispherical reflectance graphs derived from the round robin participants' measurement data. The need for post-processing of data becomes visible as the signal continues showing around $10 \%$ of deviation to the other institutes' data.
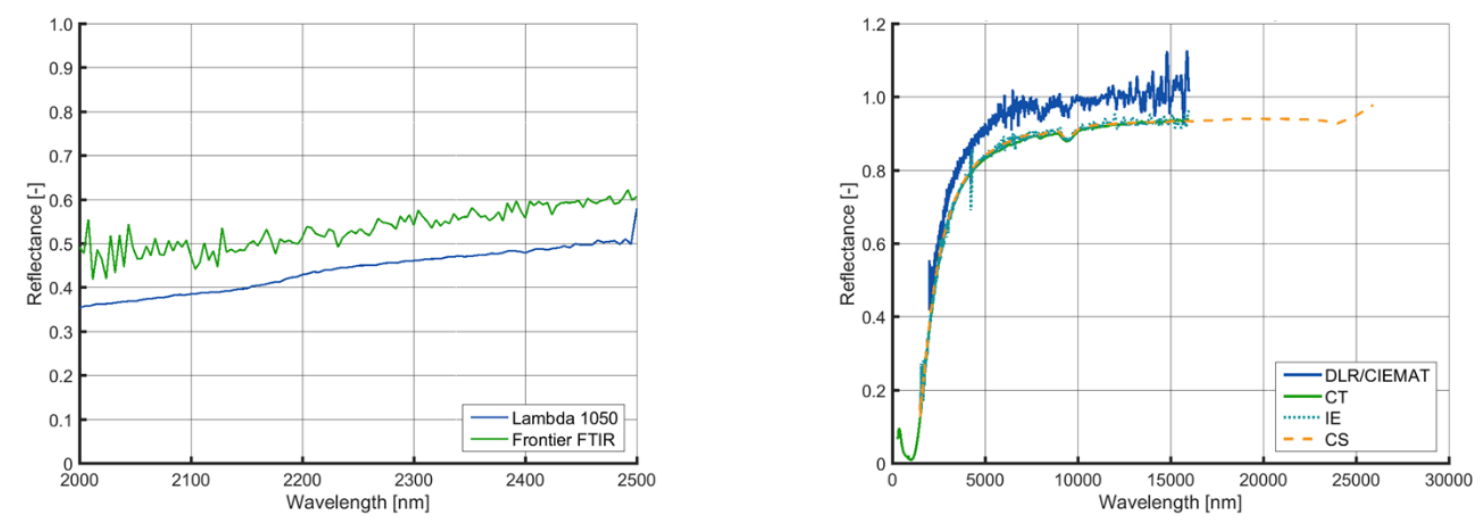

Figure 1 - Internal measurement deviations at DLR/CIEMAT [8]; left: mismatch between two complementary spectrophotometers; right: comparison of initial measurement data with round robin data.

The approach worked out for the mismatch correction bases on the common wavelength interval of the Lambda 1050 and the Frontier FTIR spectrophotometer. The deviation $\rho_{\text {offset }}$ between the two signals is determined as described in Eq. 5. The calculation is based on the common wavelength interval between 2,000 and 2,500 nm and considered after interpolation in steps of $1 \mathrm{~nm}$. Hence, 500 data points are available [8].

$$
\rho_{\text {offset }}(\lambda)=\rho_{\text {meas }, F T I R}(\lambda)-\rho_{\text {hem }, 1050}(\lambda)
$$

The mean deviation $\rho_{\text {offset,avg }}$ is determined according to Eq. 6.

$$
\rho_{\text {offset, avg }}=\frac{1}{500} * \sum_{1}^{500} \rho_{\text {offset }}(\lambda)
$$

Eq. 6

For the correction of the whole Frontier FTIR measurement range, the mean offset is considered constant up to $16 \mu \mathrm{m}$ and the raw Frontier FTIR measurement values $\rho_{\text {meas,FTIR }}(\lambda)$ are corrected to $\rho_{\text {hem,corr }}(\lambda)$ as indicated in Eq. 7 [8].

$$
\rho_{\text {hem,corr }}(\lambda)=\rho_{\text {meas, FTIR }}(\lambda)-\rho_{\text {offset, avrg }}
$$

The result is shown in Figure 2 (right). This calculation approach permits the post-processing of the raw Frontier FTIR spectral hemispherical reflectance measurement data for all samples that have been characterised with the Lambda 1050 spectrophotometer as well. For future measurements, a new measurement routine with new reference standards promises the correction of the spectral mismatch without the need for post-processing. Consequently, Frontier FTIR data can be determined independently from measuring the sample with the Lambda 1050 spectrophotometer. 


\section{Initial Round Robin Data}

Figure 2 shows the spectral hemispherical reflectance values measured by the different laboratories.
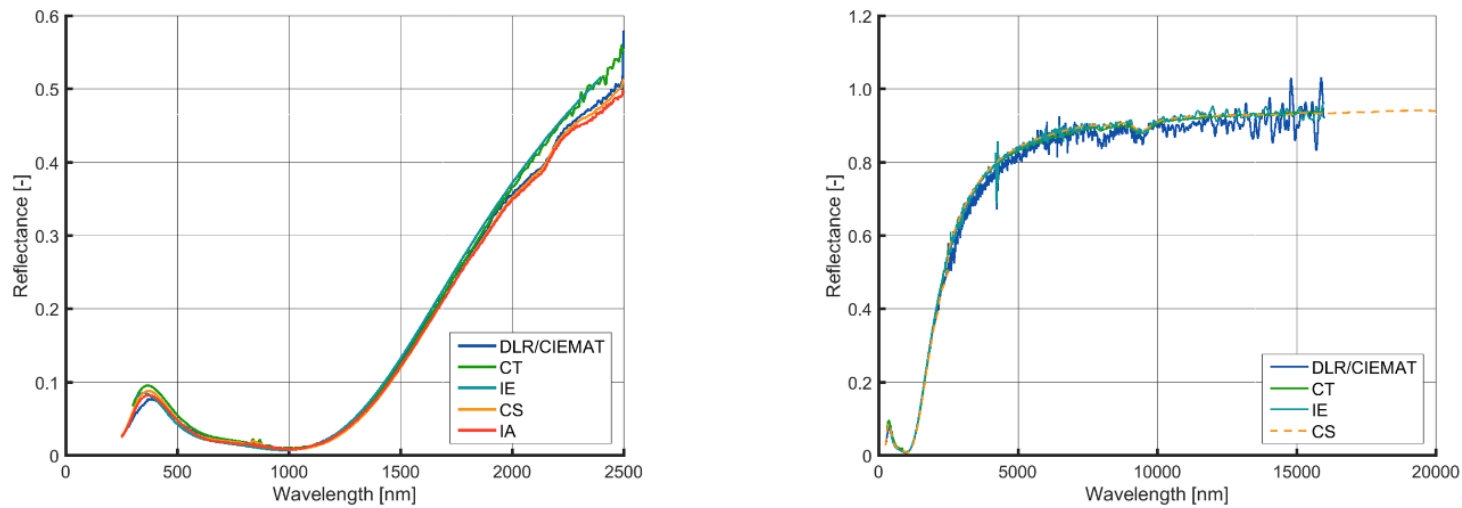

Figure 2 - Spectral hemispherical reflectance measurement values as provided by the partner institutes [8]. Left: Short wavelength range from 280 to $2,500 \mathrm{~nm}$ as used for solar absorptance calculation. Right: Broad wavelength range from 280 to $16,000 \mathrm{~nm}$ as used for thermal emittance calculation (DLR/CIEMAT data mismatch corrected).

Solar absorptance values initially provided by the participating laboratories are shown in Table 3 . They range from $93.9 \%$ to $94.6 \%$, the standard deviation (stdev) is $0.29 \%$. This small deviation indicates a reproducible measurement process. Figure 2 (left) shows overlapping spectra in the range from 250 to 2,500 nm, although small deviations are visible in the range from 2,000 to $2,500 \mathrm{~nm}$. Calculated solar absorptance values may deviate because of measurement deviations or different calculation approaches.

Table 3 - Initial solar absorptance values as provided by the round robin participating institutes [8]

\begin{tabular}{|c|c|c|c|c|c||c|c|}
\hline & CT & IE & CS & $\begin{array}{c}\text { DLR/ } \\
\text { CIEMAT }\end{array}$ & IA & avg & stdev \\
\hline $\boldsymbol{\alpha}_{\text {s initial [\%] }}$ & 93.9 & 94.6 & 94.4 & 94.4 & 93.9 & 94.2 & 0.29 \\
\hline
\end{tabular}

Thermal emittance values provided for the round robin campaign show higher deviations. Table 4 reveals a range from $8.8 \%$ to $25.4 \%$. No DLR/CIEMAT data is included at this point due to the internal measurement mismatch that needs to be corrected first. IA's value cannot be included in the analysis as no spectral data is provided by the portable device (see Table 2). The standard deviation within the other three institute's data is determined to be $0.69 \%$, although Figure 2 (right) shows overlapping spectra. Consequently, the reason for the deviation is searched in the processing of spectral data.

Table 4 - Initial thermal emittance round robin values as provided by the participating institutes [8]

\begin{tabular}{|c|c|c|c|c|c||c|c|}
\hline & CT & IE & CS & $\begin{array}{c}\text { DLR/ } \\
\text { CIEMAT }\end{array}$ & IA & avg & stdev \\
\hline $\boldsymbol{\varepsilon}_{\text {th }}$ initial [\%] & 25.4 & 23.9 & 25.3 & - & 8.8 & 24.9 & 0.69 \\
\hline
\end{tabular}

The analysis of the partner institutes' calculation methods reveals the need for common processing of spectral data. It can be observed that measurement resolutions vary from 1 to $10 \mathrm{~nm}$ in the short wavelength range and up to over 150 $\mathrm{nm}$ in the infrared (IR) range. Additionally, the wavelength limits in consideration differ. The lower limit $\lambda_{1}$ is found to be between 250 and $300 \mathrm{~nm}$ for the different institutes, while the upper limit $\lambda_{3}$ deviates between 16 and $50 \mu \mathrm{m}$. 
This indicates that some institutes already carried out extrapolation while others calculated within the limits of their measurement ranges.

Furthermore, the examination of the calculation processes shows that the partner institute IA used other reference spectra, for solar absorptance as well as for thermal emittance (see Table 2). The solar reference spectrum has been considered extra-terrestrial with AM 0 and thermal emittance has been calculated with a black body reference of 300 $\mathrm{K}\left(27^{\circ} \mathrm{C}\right)$ while all other institutes calculated with $923 \mathrm{~K}\left(650^{\circ} \mathrm{C}\right)$. The latter serves as explanation for the low thermal emittance value of $8.8 \%$ as thermal emittance decreases at lower temperatures.

Table 5 shows the coatings thermal efficiency as calculated from the solar absorptance and thermal emittance values provided by the round robin participants. DLR/CIEMAT is not included due to the primary need for mismatch correction. Furthermore, the falsified IA value is excluded. The data from the remaining institutes permits the determination of a mean thermal efficiency of $90.81 \%$ with a standard deviation of $0.37 \%$.

Table 5 - Thermal efficiency as calculated from the initial solar absorptance and thermal emittance values

\begin{tabular}{|c|c|c|c|c|c||c|c|}
\hline & CT & IE & CS & $\begin{array}{c}\text { DLR/ } \\
\text { CIEMAT }\end{array}$ & IA & avg & stdev \\
\hline$\eta_{\text {coating initial [\%] }}$ & 90.33 & 91.24 & 90.85 & - & - & 90.81 & 0.37 \\
\hline
\end{tabular}

\section{CALCULATION METHOD}

A new proposal for the processing of spectral hemispherical reflectance values for the determination of solar absorptance and thermal emittance is worked out. The aspects found in the analysis of initial measurement values and calculations are examined.

\section{Weighting normalisation}

As the participants use different reference spectra, common reference spectra need to be defined first. Figure 3 shows the reference spectra. For solar absorptance, this indicates the use of the ASTM G173-03 direct solar spectrum at AM 1.5 as IA was found to have used AM 0. In the case of thermal emittance, the reference spectrum is Planck's Law as shown in Eq. 3 with a reference temperature of $923 \mathrm{~K}\left(650^{\circ} \mathrm{C}\right)$. Physical constants used in the equation are defined according to CODATA 2014 [6].
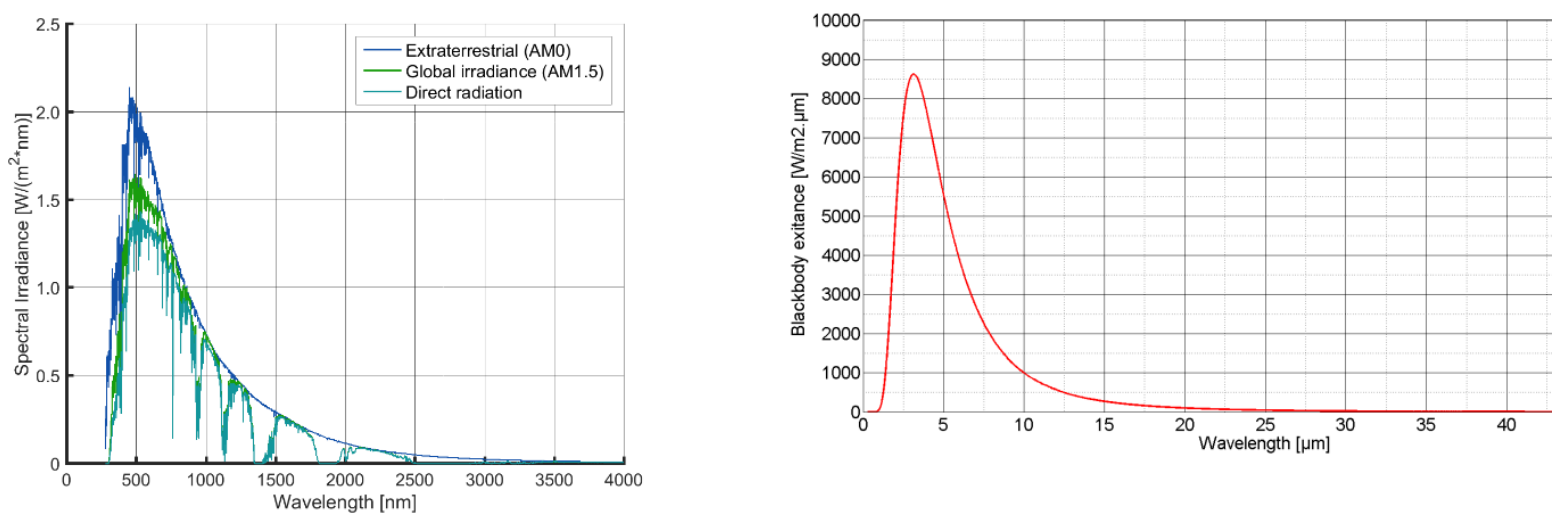

Figure 3 - Reference spectra [8]; left: ASTM G173-03 as used for solar absorptance; right: ideal black body exitance at $923 \mathrm{~K}$ as used for thermal emittance 


\section{Extrapolation}

To ensure the consideration of the same wavelength range for all datasets, extrapolation needs to be carried out. It enables the analysis of the influence of the variation of the upper wavelength limit $\lambda_{3}$ on the result of thermal emittance and hence a consideration of upper wavelength limits greater than the measurement intervals. Three approaches are compared:

1. MATLAB default linear extrapolation algorithm. Last available data interval is considered constant.

2. Approach IE as used by one partner institute already. The mean spectral hemispherical reflectance in the IR range is determined between 12 and $14 \mu \mathrm{m}$ and then considered constant.

3. Approach ISO as proposed in DIN EN ISO 22975-3 [9]. The spectral hemispherical reflectance determined for the upper wavelength limit of the spectrophotometer is considered constant.

As proposed in DIN EN ISO 22975-3, the upper wavelength limit is set to $50 \mu \mathrm{m}$. Figure 4 shows a graphic overview of the extrapolation approaches and aims at verifying them with the measurement data from the project partner CS which is available for wavelength ranges up to $25 \mu \mathrm{m}$. It reveals that the MATLAB algorithm is not suitable for the extrapolation of spectral hemispherical reflectance data as it relies on the last wavelength interval available and considers it linear. The extrapolation approaches IE and ISO show a small deviation between themselves and agree to the reference data. Hence, both approaches are considered applicable for a selective coating. In the following calculations, the approach according to DIN EN ISO 22975-3 is used.

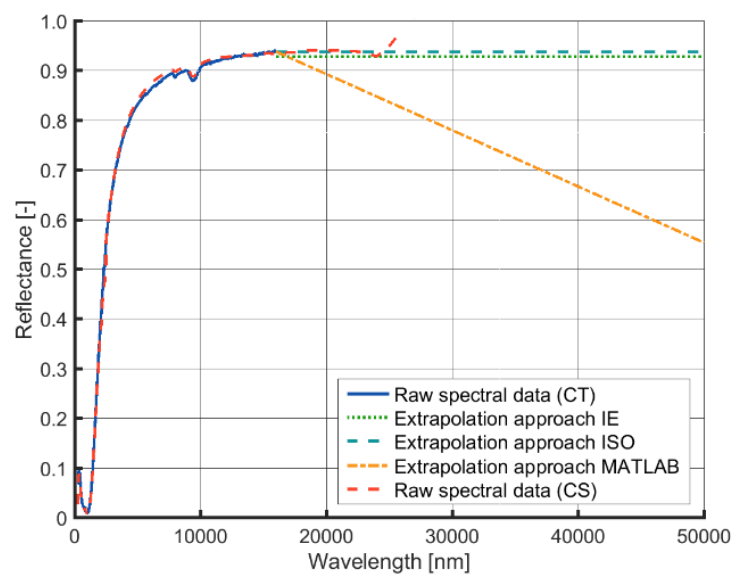

Figure 4 - Comparison of extrapolation approaches and validation with measurement data [8]

\section{Wavelength range}

For the determination of solar absorptance and thermal emittance, variation of the lower wavelength limit $\lambda_{1}$ is carried out. The influence is to be evaluated between the limits of 250 and $300 \mathrm{~nm}$, as these are the values provided by round robin partner institutes. After the extrapolation of spectral hemispherical reflectance up to $50 \mu \mathrm{m}$, the influence of a variation of the upper wavelength limit on thermal emittance is examined as well. $\lambda_{3}$ values used by the partner institutes are 16, 25 and $50 \mu \mathrm{m}$.

It is found that the variation of $\lambda_{1}$ between 250 and $300 \mathrm{~nm}$ does not result in a significant change for the solar absorptance or thermal emittance values. Table 6 shows the results achieved with interpolation, common reference spectra and a common wavelength range. $\lambda_{1}$ is defined as $280 \mathrm{~nm}$. Solar absorptance is considered up to $\lambda_{2}$ of 2,500 $\mathrm{nm}$ and thermal emittance up to $\lambda_{3}$ of $16 \mu \mathrm{m}$ (no extrapolation). Standard deviations can be reduced to $0.2 \%$ for solar absorptance and $0.16 \%$ for thermal emittance (DLR/CIEMAT not considered for the statistics). 
Table 6 - Solar absorptance and thermal emittance (923 K) after weighting normalisation and with common $\lambda_{1}$ [8]

\begin{tabular}{|c|c|c|c|c|c||c|}
\hline & CT & IE & CS & $\begin{array}{c}\text { DLR/ } \\
\text { CIEMAT }\end{array}$ & IA & stdev \\
\hline $\boldsymbol{\alpha}_{\mathbf{S}}[\%]$ & 93.95 & 94.45 & 94.40 & 94.44 & 94.51 & 0.20 \\
\hline $\boldsymbol{\varepsilon}_{\text {th }}[\boldsymbol{\%}]$ & 25.38 & 25.02 & 25.07 & 27.22 & - & 0.16 \\
\hline
\end{tabular}

As the variation of $\lambda_{1}$ does not lead to a deviation of the result, it is considered negligible. Following, the variation of $\lambda_{3}$ is analysed. Figure 5 shows thermal emittance values at $923 \mathrm{~K}$ for wavelength intervals from $\lambda_{1}=280 \mathrm{~nm}$ to $\lambda_{3}$ of 16,25 and $50 \mu \mathrm{m}$ respectively. It reveals a trend towards lower result values for increasing wavelength intervals. At $50 \mu \mathrm{m}$, the average thermal emittance value is $24.54 \%$ and the standard deviation $0.18 \%$ (DLR/CIEMAT not considered for the statistics).

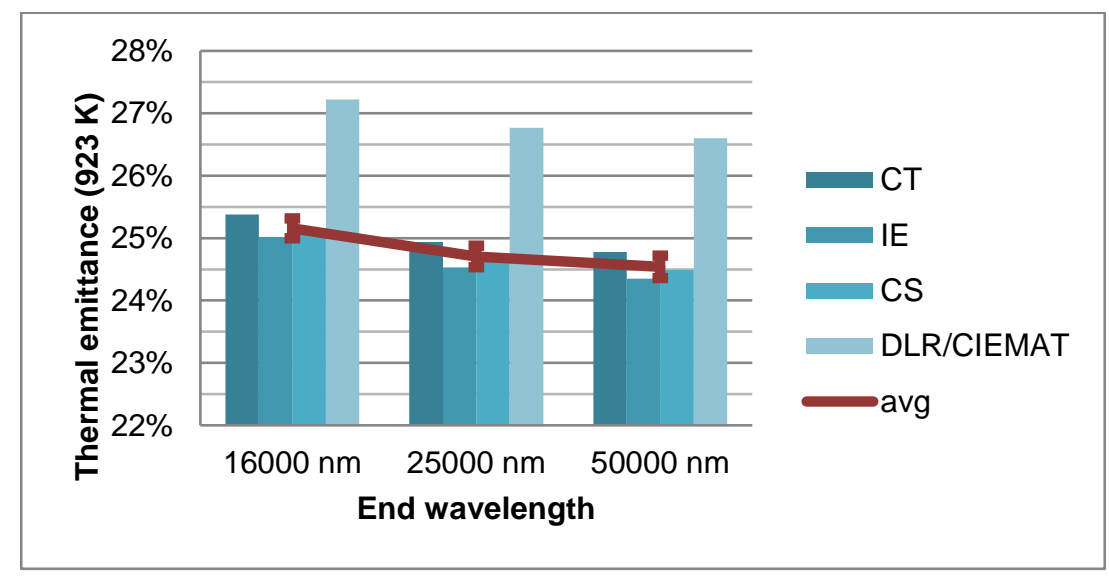

Figure 5 - Thermal emittance at different upper wavelength limits [8]

Figure 6 hints at the reason for the resulting deviation of thermal emittance when varying $\lambda_{3}$ while there is no deviation when changing $\lambda_{1}$. It shows the share of exitance of an ideal black body reached at the different wavelengths calculated as presented in Eq. 8 [8].

$$
\sigma \%(\lambda)=\frac{\sum_{\lambda_{1}}^{\lambda_{2}} E_{B B, \text { institute }}(\lambda, T)}{\int_{0}^{\infty} E_{B B}(\lambda, T)}
$$

Figure 6 (left) shows $\sigma \%$ constant at $0 \%$ for wavelengths below $1,000 \mathrm{~nm}$ which means that black body radiation is negligible at a temperature of $650{ }^{\circ} \mathrm{C}$. Hence, there is no deviation in thermal emittance when varying $\lambda_{1}$ between 250 and $300 \mathrm{~nm}$. The ASTM G173-03 reference spectrum for solar absorptance shows a similar course at small wavelengths which explains the values obtained for solar absorptance.

At wavelengths greater $10 \mu \mathrm{m}$ (Figure 6, right) there is an increase in $\sigma \%$. At $16 \mu \mathrm{m}, 96.77 \%$ of ideal black body exitance is covered by the calculation while at $50 \mu \mathrm{m}$, this value increases up to $99.86 \%$. Consequently, the calculation of thermal emittance with a wavelength range up to $50 \mu \mathrm{m}$ is recommended as a higher share of an ideal black body's radiation at $923 \mathrm{~K}$ is considered. Furthermore, this interval is proposed in DIN EN ISO 22975-3. 

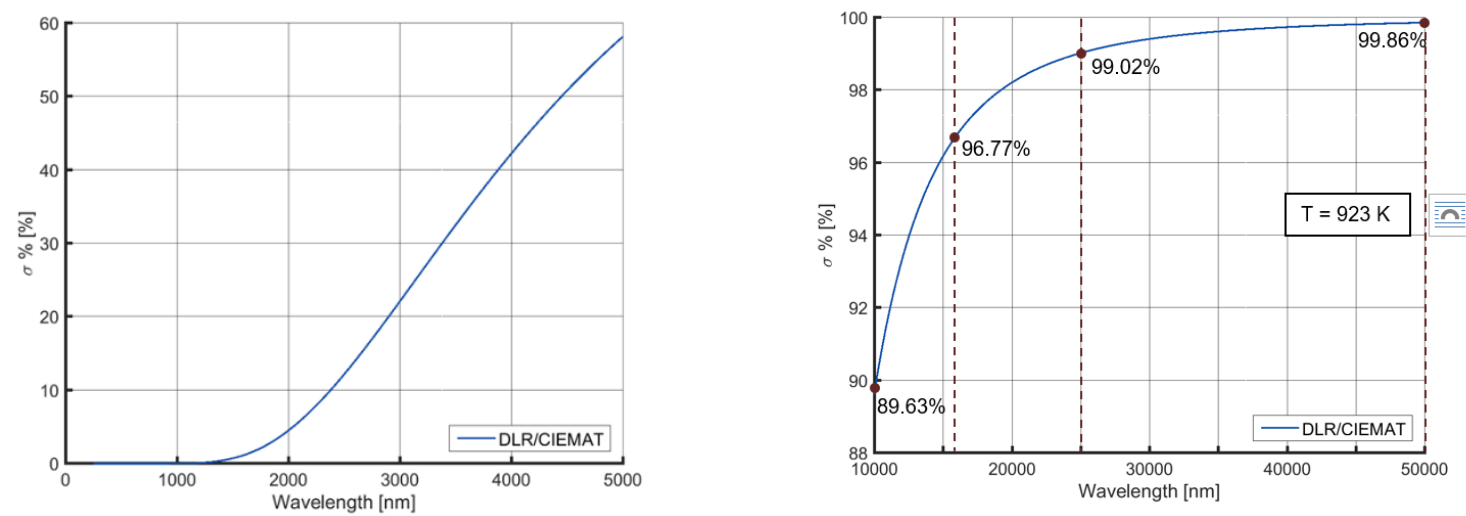

Figure 6 - Black body exitance at $923 \mathrm{~K}$ [8]; left: wavelength range 0 to $5,000 \mathrm{~nm}$; right: 10,000 to $50,000 \mathrm{~nm}$

Higher wavelength ranges lead to smaller thermal emittance values as for selective absorber coatings great wavelengths coincide with high values for spectral hemispherical reflectance and high values of $\rho_{\text {hem }}(\lambda)$ in Eq. 2 lead to smaller result values.

\section{RESULTS AND DISCUSSION}

The processing steps presented in the course of this paper are used to re-calculate optical properties from the spectral values provided in the course of the round robin campaign. Table 7 compares solar absorptance and thermal emittance values without and with common processing of spectral hemispherical reflectance data. Values without processing refer to the initial values provided by the participating laboratories such as presented in Table 3 and Table 4. The values with common processing are obtained by performing the calculation steps presented. IA values are not included as no spectral data is available. DLR/CIEMAT is not included either due to the need of post-processing of data in order to correct spectral mismatch.

Table 7 - Solar absorptance, thermal emittance and thermal efficiency before and after common data processing

\begin{tabular}{|c|c|c|c|c|}
\hline & \multicolumn{2}{|c|}{ Without common processing } & \multicolumn{2}{c|}{ With common processing } \\
\hline & avg & sdtev & avg & stdev \\
\hline $\boldsymbol{\alpha}_{\mathrm{s}}[\%]$ & 94.20 & 0.29 & 94.35 & 0.20 \\
\hline $\boldsymbol{E}_{\text {th }}[\%]$ & 24.87 & 0.69 & 24.54 & 0.18 \\
\hline $\boldsymbol{\eta}_{\text {coating [\%] }}$ & 90.81 & 0.37 & 90.82 & 0.25 \\
\hline
\end{tabular}

Average solar absorptance increased slightly through common processing. The mean value obtained is determined to be $94.35 \%$ with a reduced standard deviation of $0.20 \%$. Thermal emittance at $923 \mathrm{~K}$ decreased with common spectral data processing. Standard deviation of the three institutes considered was almost divided by a factor 4, from $0.69 \%$ to $0.18 \%$. The post-processing approach for DLR/CIEMAT data presented in Eq. 5 to Eq. 7 enables the determination of thermal emittance with a systematic deviation of $+2.06 \%$ from the average value after introducing the common measurement routine. The standard deviation of thermal efficiency determined with the commonly processed values was reduced to $0.25 \%$. Figure 7 sums up the performed processing steps. 


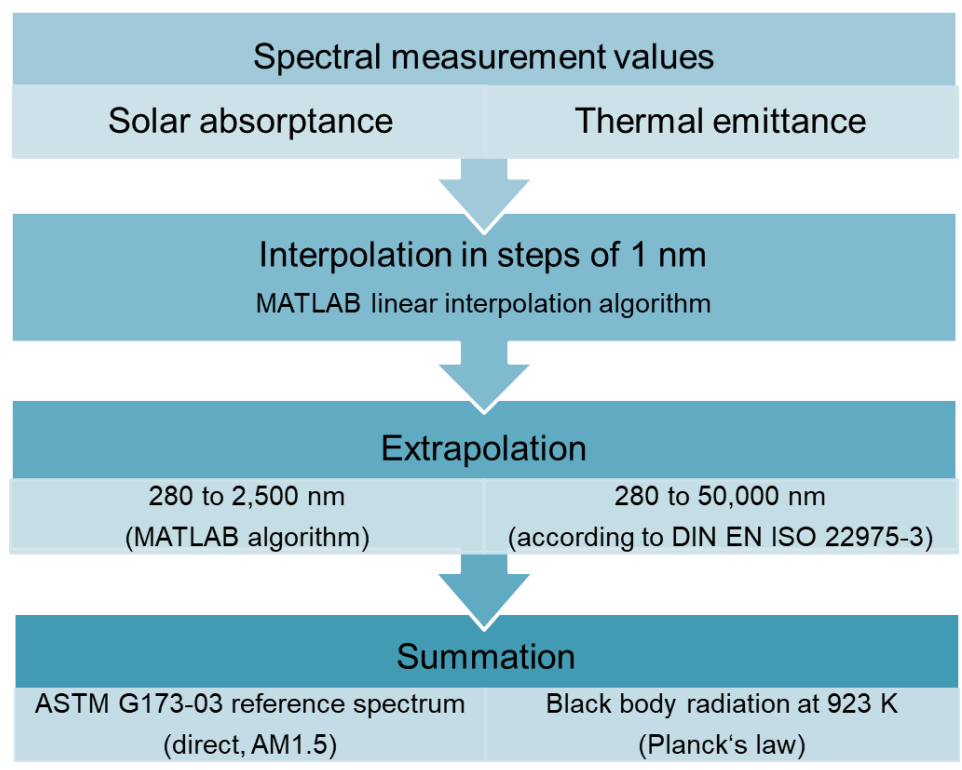

Figure 7 - Recommended processing steps for the determination of solar absorptance (left column) and thermal emittance (right column)

Solar absorptance and thermal emittance are determined according to DIN EN ISO 22975-3. Spectral values are interpolated linearly in steps of $1 \mathrm{~nm}$. The wavelength interval in consideration ranges from 280 to $2,500 \mathrm{~nm}$ for solar absorptance and from 280 to $50,000 \mathrm{~nm}$ for thermal emittance. Reference spectra are the ASTM G173-03 direct AM1.5 solar spectrum and the ideal black body exitance according to Planck's Law at $923 \mathrm{~K}$ with the use of physical constants according to CODATA 2014 [6].

The extrapolation approach according to DIN EN ISO 22975-3 provides satisfactory results for selective samples. It needs to be verified if the same approach is still suitable for black absorber coatings. Black coatings with high thermal emittance do not show constant high reflectance values in the IR range. Hence, considering the last value available at $16 \mu \mathrm{m}$ might lead to high deviations in the extrapolation step. Also, measurement errors in the IR range could bias the spectral hemispherical reflectance values obtained from the DIN EN ISO 22975-3 extrapolation approach.

\section{CONCLUSION}

The implementation of a standard calculation procedure for the calculation of solar absorptance and thermal emittance from spectral hemispherical reflectance values permits the optical characterisation of a flat sample with a selective solar absorber coating. Optical properties are determined with standard deviations of $0.2 \%$. Reducing the standard deviations for solar absorptance as well as for thermal emittance determined with the initial spectral hemispherical reflectance values provided by the round robin participants is considered as verification of measurement reproducibility, according to ASTM E 691 - 05 [10]. The more certain determination of solar absorptance and thermal emittance enable reducing the standard deviation of thermal efficiency to $0.25 \%$.

Achieving a reduction of standard deviation of thermal emittance of a factor $\mathrm{x} 4$ points out the need for common processing of measurement data in interlaboratory studies. The definition of reference spectra and wavelength ranges is highly recommendable when characterising samples in different laboratories.

Additionally, the internal measurement error at DLR/CIEMAT was corrected and a new measurement routine was enabled. Further measurements are supposed to enlarge the number of institutes included in the Raiselife round robin campaign. 


\section{ACKNOWLEDGMENTS}

Financial support from the European Union is gratefully acknowledged (EU-Raiselife project, Horizon 2020, Contract $\left.\mathrm{n}^{\circ} 686008\right)$.

\section{REFERENCES}

[1] Sutter, F., „Raising the Lifetime of Functional Materials for Concentrated Solar Power Technology,“ [Online]. Available: https://www.raiselife.eu/activities/index.php. [Zugriff am 2519 2017].

[2] European Comission, „What is Horizon 2020?,“ [Online]. Available: https://ec.europa.eu/programmes/horizon2020/en/what-horizon-2020. [Zugriff am 2509 2017].

[3] Caron, S. et al., „Accelerated Ageing of Solar Receiver Coatings: Experimental Results for T91 and VM12 Steel Substrates," in SolarPACES 2017, Santiago de Chile, 2017.

[4] National Renewable Energy Laboratory (NREL), „Reference Solar Spectral Irradiance: ASTM G-173,“ [Online]. Available: http://rredc.nrel.gov/solar/spectra/am1.5/astmg173/astmg173.html. [Zugriff am 0612 2017].

[5] J. A. e. a. Duffie, Solar engineering of thermal processes, Hoboken, New Jersey: John Wiley \& Sons, Inc., 2006.

[6] National Institute of Standards and Technology (NIST), „The NIST Reference on Constats, Units, and Uncertainty,“25 06 15. [Online]. Available: https://physics.nist.gov/cuu/Constants/. [Zugriff am 0712 17].

[7] ASTM International, „E 903 - 96: Standard Test Method for Solar Absorptance, Reflectance, and Transmittance of Materials Using Integrating Sphere,“ ASTM International, West Conshohocken, 1996.

[8] Herding, L., „Spectral characterisation of high temperature solar absorber coatings. Student Thesis,“ Tabernas, Spain, 2018.

[9] International Organization for Standardization, „DIN EN 22795-3: Solar energy - Collector components and materials - Part 3: Absorber surface durability, “International Organization for Standardization, Geneva, 2014.

[10] ASTM International, „E 691 - 05: Standard Practice for Conducting an Interlaboratory Study to Determine the Precision of a Test Method,“ ASTM International, West Conshohocken, 2005. 